\title{
ANÁLISE DO PERFIL EPIDEMIOLÓGICO E TENDÊNCIA TEMPORAL DOS ACIDENTES GRAVES DE TRABALHO
}

\author{
ANALYSIS OF THE EPIDEMIOLOGICAL PROFILE \\ AND TEMPORAL TREND OF SERIOUS \\ OCCUPATIONAL ACCIDENTS
}

\section{ANÁLISIS DEL PERFIL EPIDEMIOLÓGICO Y LA TENDENCIA TEMPORAL DE ACCIDENTES DE TRABAJO GRAVES}

\author{
Ana Caroline Rodrigues Lima ${ }^{1}$ \\ Damião da Conceição Araújoº \\ Jadiel Fellipe Santana Santos ${ }^{3}$ \\ Jéssica Oliveira da Cunha ${ }^{4}$ \\ Julianne Souza Bezerra 5 \\ Alan Dantas dos Santos ${ }^{6}$
}

Como citar este artigo: Lima ACR, Araújo DC, Santos JFS, Cunha JO, Bezerra JS, Santos AD. Análise do perfil epidemiológico e tendência temporal dos acidentes graves de trabalho. Rev baiana enferm. 2019;33:e33230.

Objetivo: analisar o perfil epidemiológico e a tendência temporal dos acidentes graves de trabalho no estado de Sergipe, no período de 2009 a 2015. Método: estudo ecológico, descritivo, de série temporal, com base em dados secundários informados no Sistema de Informação de Agravos de Notificação. Resultados: foram notificados 1.271 acidentes graves de trabalho com trabalhadores entre 16 e 65 anos de idade; os anos de 2011 (19,5\%) e 2009 (15,6\%) foram os de maior número de registros; 2011 (23,6\%) e 2010 (19.9\%) apresentaram maior incidência; 2014 e 2015 registraram as menores taxas. Perfil dos trabalhadores acidentados: sexo masculino (88,8\%), entre 25 e 29 anos (17,1\%), pardos (61,5\%), ensino incompleto da $5^{\underline{a}}$ a $8^{\underline{a}}$ série (20,2\%). Conclusão: a análise do perfil epidemiológico e a tendência temporal dos acidentes graves de trabalho no estado de Sergipe revelou tendência geral decrescente no período analisado, ainda que fosse elevado o número de acidentes registrados.

Descritores: Epidemiologia Descritiva. Sistemas de Informação. Saúde do Trabalhador. Acidentes de Trabalho.

Objective: to analyze the epidemiological profile and the temporal trend of serious occupational accidents in Sergipe, in the period from 2009 to 2015. Method: ecological, descriptive, time-series study, based on secondary data reported on the Information System for Disease Notification. Results: there were 1,271 reports of serious occupational accidents with workers aged between 16 and 65 years; the years 2011 (19.5\%) and 2009 (15.6\%) presented the highest number

\footnotetext{
Enfermeira. Mestre em Biologia Parasitária. Professora da Universidade Federal de Sergipe. Lagarto, Sergipe, Brasil. http://orcid.org/0000-0002-9I09-2435

2 Enfermeiro. Mestre em Biologia Parasitária. Universidade Federal de Sergipe. Lagarto, Sergipe, Brasil. http://orcid.org/0000-0003-I I I6- I70X

Enfermeiro. Especialista em Saúde da Família. Enfermeiro assistencial pela Empresa Brasileira de Serviços Hospitalares. Aracaju, Sergipe, Brasil. http://orcid.org/0000$0003-4324-513$

4 Enfermeira. Especialista em Saúde da Família. Universidade Federal de Sergipe. São Cristóvão, Sergipe, Brasil. jessicaocunha@hotmail.com http://orcid.org/00000003-3670-9524

Estudante de Enfermagem. Universidade Federal de Sergipe. Lagarto, Sergipe, Brasil. jubezerra.ufs@gmail.com https://orcid.org/0000-0002-5 |44-0984

6 Enfermeiro. Doutor em Ciências da Saúde. Professor da Universidade Federal de Sergipe. Lagarto, Sergipe, Brasil. http://orcid.org/0000-0002-6529- | 887
} 
of records; 2011 (23.6\%) and 2010 (19.9\%) had the highest incidence; 2014 and 2015 recorded the lowest rates. Profile of injured workers: males (88.8\%), between 25 and 29 years (17.1\%), pardos (61.5\%), incomplete education from $5^{\text {th }}$ to $8^{\text {th }}$ grade $(20.2 \%)$. Conclusion: the analysis of the epidemiological profile and the temporal trend of serious occupational accidents in the state of Sergipe showed decreasing trend in the analyzed period, although the number of accidents recorded was high.

Descriptors: Epidemiology, Descriptive. Information Systems. Occupational Health. Accidents, Occupational.

Objetivo: analizar el perfil epidemiológico y la tendencia temporal de los accidentes de trabajo graves en el estado de Sergipe, en el período comprendido entre 2009 y 2015. Método: estudio ecológico, descriptivo, de series de tiempo, basado en datos secundarios reportados en el Sistema de Información de Agravios de Notificación. Resultados: se reportaron 1.271 accidentes de trabajo graves con los trabajadores con edad entre 16 y 65 años; los años 2011 (19,5\%) y 2009 (15,6\%) fueron el mayor número de registros; 2011 (23,6\%) y 2010 (19,9\%) tuvieron una mayor incidencia; 2014 y 2015 registraron las tasas más bajas. El perfil de los trabajadores lesionados: varones (88,8\%), entre 25 y 29 años (17,1\%), pardos (61,5\%), enseñanza incompleta del 5o a 8o grado (20,2\%). Conclusión: el análisis del perfil epidemiológico y la tendencia temporal de accidentes de trabajo graves en el estado de Sergipe mostró una tendencia decreciente en el período analizado, aunque el número de accidentes registrados fue elevado.

Descriptores: Epidemiología Descriptiva. Sistemas de Información. Salud Laboral. Accidentes de Trabajo.

\section{Introdução}

O trabalho representa uma dimensão fundamental na estruturação do indivíduo, da família e da sociedade, no que se refere ao desenvolvimento da capacidade laboral e à garantia das condições materiais para sobrevivência. No entanto, ao longo dos tempos, tornou-se um processo provocador de sofrimentos, adoecimentos e morte. As doenças/agravos ocupacionais advindos da execução do trabalho podem ser consequência da exposição dos profissionais a riscos biológicos, físicos, químicos, psicossociais e ergonômicos ${ }^{(1-2)}$.

Os trabalhadores adoecem e morrem por causas relacionadas ao trabalho como consequência direta das atividades profissionais que exercem ou exerceram, ou pelas condições adversas em que seu trabalho é ou foi realizado ${ }^{(3)}$.

Dessa forma, o trabalho impacta diretamente sobre o perfil de morbimortalidade dos trabalhadores, seja de forma direta - com os acidentes de trabalho e as doenças profissionais -, seja de forma indireta - doenças relacionadas com o trabalho. A consequência direta das atividades profissionais que os trabalhadores exercem ou exerceram em seu ambiente de trabalho ou as condições adversas em que o trabalho é ou foi realizado favorecem o processo de adoecimento e morte ${ }^{(4)}$.
O acidente de trabalho pode ser definido como um evento ocorrido no exercício de atividade laborativa, independentemente da situação empregatícia e previdenciária do trabalhador, que acarreta danos potenciais à saúde, de forma temporária ou permanente, desencadeando lesão corporal e, como consequência, perda ou redução da capacidade para o trabalho ${ }^{(5)}$.

Legalmente, os acidentes de trabalho podem ser classificados em: os que ocorrem no ambiente de trabalho, e os de trajeto ${ }^{(6)}$. O primeiro é definido como o que ocorre dentro do ambiente de trabalho e no seu exercício; o de trajeto é conceituado como o acidente sofrido fora do local e do horário de trabalho, que ocorre durante o percurso para a residência ou vice-versa, por qualquer meio de locomoção ${ }^{(7)}$. Dentre os tipos de acidentes de trabalho destacam-se aqueles classificados como graves, que implicam em comprometimentos físicos, funcionais e até mesmo resultados mais fatais, como a mutilação ${ }^{(8)}$.

Para a saúde pública, os acidentes laborais são agravos que comprometem significativamente as condições de vida e de saúde da população no provimento do sustento dos núcleos familiares e no movimento da economia de um país ${ }^{(9)}$. 
O presente estudo justifica-se diante da compreensão de aspectos como: o quantitativo de trabalhadores que foram vítimas de acidentes graves é significativo no estado de Sergipe, com 1.271 casos notificados no período de 2009 a 2015; as ações de saúde do trabalhador devem ser norteadas utilizando-se o perfil epidemiológico; os estudos que abordam tendência temporal permitem o conhecimento da distribuição e as características dos acidentes que ocorreram em um determinado período; são poucos os estudos no estado de Sergipe que abordam a temática.

No mundo, ocorrem anualmente mais de 2 milhões de óbitos devido ao trabalho, com representatividade de 318 mil mortes em decorrência dos acidentes e 2 milhões em detrimento de doenças relacionadas ao trabalho ${ }^{(10)}$. Os acidentes de trabalho são responsáveis por 18\% das mortes em países de rendas média e baixa; já em países de alta renda, esse percentual é de apenas 5\%.

O cenário demonstra o amplo custo no que se refere à morbimortalidade provocada pelo trabalho, com o impacto maior nos países de baixa e média renda, onde grande parte da população está envolvida em atividades perigosas, tais como mineração. No Brasil, os custos com os acidentes e doenças do trabalho ultrapassam R $\$ 70$ bilhões ao ano, o que corresponde a aproximadamente 9\% da folha salarial do país. Para as famílias, os custos e os danos aos trabalhadores são estimados em R $\$ 16$ bilhões. Entretanto, esse custo é subestimado, pois se refere apenas ao setor formal do mercado de trabalho ${ }^{(11)}$.

Os agravos da saúde do trabalhador constituem uma temática que apresenta dimensão social de suma importância para a saúde pública, e vêm, cada vez mais, desafiando a atuação do estado, para que mais intervenções sejam realizadas e tornem-se mais frequentes, para que se consiga reduzir os custos da seguridade social do país ${ }^{(12-13)}$.

Frente às considerações, o objetivo deste estudo é analisar o perfil epidemiológico e a tendência temporal dos acidentes graves de trabalho no estado de Sergipe, no período de 2009 a 2015.

\section{Método}

Trata-se de um estudo ecológico, descritivo, de série temporal com base em dados secundários notificados no Sistema de Informação de Agravos de Notificação (SINAN) alimentado pelo DATASUS da Secretaria Estadual de Saúde de Sergipe. Analisou-se a série histórica (2009 a 2015) da incidência de Acidentes Graves de Trabalho ocorridos nos municípios do estado de Sergipe. O período escolhido para o estudo decorreu da disponibilidade dos dados no sistema. O estado de Sergipe localiza-se no litoral do Nordeste do Brasil, conta com uma população de 2.068.017 hab. $/ \mathrm{km}^{2}$, e uma área de $21.910,354 \mathrm{~km}{ }^{2}$, equivalente a $0,26 \%$ de todo o território nacional ${ }^{(14)}$.

A totalidade dos acidentes de trabalhos considerados graves, registrados no Sinan entre os anos 2007 e 2010, com os trabalhadores de idade acima de 16 anos e abaixo de 65 anos, foi incluída no estudo, uma vez que, para o cálculo da incidência, foi utilizada a População Economicamente Ativa (PEA), definida como sendo o potencial de mão de obra disponível para atuar no setor produtivo, isto é, a população ocupada e a população desocupada, com idade até 65 $\operatorname{anos}^{(15)}$.

Os dados foram coletados por meio de um instrumento estruturado, elaborado pelo pesquisador, de acordo com as varáveis de interesse para o estudo, bem como em relação à plataforma do SINAN, composto de duas partes:

a) dados sociodemográficos: sexo (masculino/feminino); cor da pele/raça (branca, preta, parda, amarela, indígena e ignorada/em branco); escolaridade (analfabeto, $1^{\underline{a}}$ a $4^{\underline{a}}$ série incompleta, $4^{\underline{a}}$ série completa, $5^{\underline{a}}$ a $8^{\underline{a}}$ série incompleta, ensino fundamental completo, ensino médio incompleto, ensino médio completo, educação superior incompleta, educação superior completa) e idade (16-19, 20-24, 25-29, 30-34, 35-39, 40-44, 45-49, 50-54, 55-59, 60-65);

b) dados para caracterização do acidente: tipo do acidente (típico, trajeto, ignorado), se houve emissão de Comunicação de Acidente de Trabalho (CAT), regime de tratamento (hospitalar, ambulatorial, ambos, ignorado) e evolução 
dos casos (cura, incapacidade temporária, incapacidade parcial, incapacidade total permanente, óbito por acidente de trabalho grave, óbito por outras causas, outro, ignorado).

Os dados foram armazenados em planilhas eletrônicas e analisados com auxílio do programa Microsoft Excel 2010. Foi realizada análise descritiva da amostra. As variáveis categóricas foram expressas por meio de frequência absoluta e relativa, e as numéricas, como a idade, foram estratificadas para serem expressas da mesma forma que as categóricas.

O cálculo das taxas de incidência anual dos acidentes graves de trabalho foi feito com a divisão do número absoluto de acidentes no ano, pela PEA do respectivo ano, obtida junto à Superintendência de Estudos e Pesquisas (SUPES) da Secretaria de Planejamento e Gestão (SEPLAG).

Analisou-se a tendência para cada região de saúde (RS) por regressão linear, e foram consideradas significativas as tendências com $\mathrm{p}<0,05$, além do valor de coeficiente de determinação (R2) e a análise dos resíduos. No modelo linear simples, as que possuíssem algum conjunto de dados com duas variáveis mensuradas como x e y, a correlação entre si indicava um comportamento linear e foi ajustada a uma reta que melhor se adequasse aos pares dos valores, utilizando-se o método denominado mínimos quadrados.

No modelo linear, para as que possuíam um conjunto de dados no qual foram medidas duas variáveis, x e y, cuja correlação entre si indicava um comportamento linear, foi ajustada uma reta que melhor se encaixasse nesses pares de valores pelo método de mínimos quadrados.

O estudo foi efetuado apenas com dados de origem secundária de acesso livre/gratuito, e os preceitos éticos foram atendidos conforme Resolução n. 466, de 12 de dezembro de 2012, do Conselho Nacional de Saúde (CNS).

\section{Resultados}

O número total de acidentes de trabalho graves notificados no estado de Sergipe, no período de 2009 a 2015, foi de 1.271 casos. Observou-se, com relação ao número de acidentes, que os dois anos de destaque foram 2011 (19,5\%) e 2009 (15,6\%); já com relação à incidência, calculada pela PEA, tiveram destaque os anos de 2011 e 2010 com as respectivas taxas de incidência de $23,6 \%$ e 19,9\%; os anos de 2014 e 2015 foram os que registram as menores taxas (Tabela 1).

Tabela 1 - Frequência e incidência de acidentes de trabalho graves ocorridos e registrados por ano. Sergipe, Brasil - 2009-2015 (N=1271)

\begin{tabular}{l|c|c|c}
\hline Ano & $\mathbf{n}$ & $\mathbf{0}$ & $\begin{array}{c}\text { Incidência } \\
\text { (por 100 mil entre a PEA) }\end{array}$ \\
\hline 2009 & 198 & 15,6 & 18,6 \\
2010 & 185 & 14,6 & 19,9 \\
2011 & 248 & 19,5 & 23,6 \\
2012 & 160 & 12,6 & 14,9 \\
2013 & 187 & 14,7 & 17,5 \\
2014 & 132 & 10,4 & 11,5 \\
2015 & 161 & 12,7 & 14,0 \\
Total & $\mathbf{1 2 7 1}$ & $\mathbf{1 0 0}$ & \\
\hline
\end{tabular}

Fonte: Elaboração própria.

Analisando-se as características sociodemográficas dos casos de acidente de trabalho grave ocorridos entre os anos de 2009 e 2015, pôde-se obter o seguinte perfil dos acidentados: trabalhadores do sexo masculino $(88,8 \%)$, com a raça/cor da pele parda (61,5\%), entre 25 e 29 anos (17,1\%), com ensino incompleto da $5^{\mathrm{a}}$ a $8^{\underline{a}}$ série $(20,2 \%)$ (Tabela 2$)$. 
Tabela 2 - Frequência de acidentes de trabalho graves, segundo sexo, cor da pele, grau de escolaridade e idade. Sergipe, Brasil - 2009-2015 (N=1271)

\begin{tabular}{|c|c|c|}
\hline Variáveis & $\mathbf{n}$ & $\%$ \\
\hline \multicolumn{3}{|l|}{ Sexo } \\
\hline Feminino & 126 & 9,8 \\
\hline Masculino & 1145 & 88,8 \\
\hline \multicolumn{3}{|l|}{ Cor da Pele } \\
\hline Branca & 249 & 19,3 \\
\hline Preta & 62 & 4,8 \\
\hline Amarela & 3 & 0,2 \\
\hline Parda & 793 & 61,5 \\
\hline Indígena & 3 & 0,2 \\
\hline Ignorado & 161 & 12,5 \\
\hline \multicolumn{3}{|l|}{ Escolaridade } \\
\hline Analfabeto & 25 & 1,9 \\
\hline $1^{\text {a }}$ a $4^{\underline{a}}$ série incompleta & 233 & 18,1 \\
\hline $4^{a}$ série completa & 74 & 5,7 \\
\hline $5^{\text {a à }} 8^{\mathrm{a}}$ série incompleta & 261 & 20,2 \\
\hline Ensino fundamental completo & 59 & 4,6 \\
\hline Ensino médio incompleto & 88 & 6,8 \\
\hline Ensino médio completo & 247 & 19,2 \\
\hline Educação superior incompleta & 29 & 2,2 \\
\hline Educação superior completa & 27 & 2,1 \\
\hline Ignorado & 228 & 17,7 \\
\hline \multicolumn{3}{|l|}{ Idade } \\
\hline $16-19$ & 80 & 6,3 \\
\hline $20-24$ & 178 & 14,0 \\
\hline $25-29$ & 217 & 17,1 \\
\hline $30-34$ & 206 & 16,2 \\
\hline $35-39$ & 184 & 14,5 \\
\hline $40-44$ & 143 & 11,3 \\
\hline $45-49$ & 130 & 10,2 \\
\hline 50-54 & 81 & 6,4 \\
\hline 55-59 & 34 & 2.7 \\
\hline $60-65$ & 18 & 1.4 \\
\hline
\end{tabular}

Fonte: Elaboração própria.

Quanto às características do acidente, 59,8\% foram típicos e $35,8 \%$ de trajeto. A CAT somente foi emitida em 9,6\% dos casos. O regime de tratamento adotado foi principalmente o hospitalar $(57,9 \%)$, seguido do regime ambulatorial, aplicado em 20,4\% dos casos. A maioria dos casos
(62,6\%) evoluiu para incapacidade temporária, enquanto em $12,2 \%$ houve a morte do trabalhador causada diretamente pelo acidente sofrido. Apenas $14,3 \%$ dos casos evoluíram para a cura (Tabela 3). 
Tabela 3 - Frequência de acidentes de trabalho graves, segundo tipo do acidente, emissão de Comunicação de Acidente de Trabalho, regime de tratamento e evolução do caso. Sergipe, Brasil - 2009-2015

\begin{tabular}{|c|c|c|c|c|c|c|c|c|c|}
\hline \multirow{2}{*}{ Variáveis } & \multirow{2}{*}{\begin{tabular}{|c|}
2009 \\
$\mathbf{n}$ \\
\end{tabular}} & \multirow{2}{*}{\begin{tabular}{c|}
2010 \\
$n$
\end{tabular}} & \multirow{2}{*}{2011} & \multirow{2}{*}{\begin{tabular}{|c|}
2012 \\
$\mathbf{n}$
\end{tabular}} & \multirow{2}{*}{\begin{tabular}{|c|}
2013 \\
$\mathbf{n}$
\end{tabular}} & \multirow{2}{*}{\begin{tabular}{|c|}
2014 \\
n
\end{tabular}} & \multirow{2}{*}{\begin{tabular}{|c|}
2015 \\
$\mathbf{n}$
\end{tabular}} & \multicolumn{2}{|c|}{ Total } \\
\hline & & & & & & & & $\mathbf{n}$ & $\%$ \\
\hline \multicolumn{10}{|l|}{ Tipo do Acidente } \\
\hline Típico & 113 & 108 & 155 & 104 & 93 & 88 & 99 & 760 & 59,8 \\
\hline Trajeto & 75 & 74 & 86 & 51 & 91 & 34 & 44 & 455 & 35,8 \\
\hline Ignorado & 10 & 3 & 7 & 5 & 3 & 10 & 18 & 56 & 4,4 \\
\hline $\begin{array}{l}\text { Emissão de Comunicação de } \\
\text { Acidente do Trabalho }\end{array}$ & 198 & 185 & 248 & 160 & 187 & 132 & 161 & 1271 & 100,0 \\
\hline $\operatorname{Sim}$ & 42 & 11 & 17 & 14 & 11 & 19 & 8 & 122 & 9,6 \\
\hline Não & 54 & 12 & 72 & 37 & 38 & 34 & 61 & 308 & 24,2 \\
\hline Não se aplica & 70 & 51 & 112 & 43 & 43 & 42 & 23 & 384 & 30,2 \\
\hline Ignorado & 32 & 111 & 47 & 66 & 95 & 37 & 69 & 457 & 36,0 \\
\hline Regime de Tratamento & 198 & 185 & 248 & 160 & 187 & 132 & 161 & 1271 & 100,0 \\
\hline Hospitalar & 101 & 87 & 202 & 100 & 78 & 82 & 86 & 736 & 57,9 \\
\hline Ambulatorial & 83 & 41 & 17 & 12 & 74 & 18 & 14 & 259 & 20,4 \\
\hline Ambos & 2 & 47 & 23 & 16 & 19 & 5 & 6 & 118 & 9,3 \\
\hline Ignorado & 12 & 10 & 6 & 32 & 16 & 27 & 55 & 158 & 12,4 \\
\hline Evolução do Caso & 198 & 185 & 248 & 160 & 187 & 132 & 161 & 1271 & 100,0 \\
\hline Cura & 6 & 17 & 49 & 38 & 17 & 25 & 30 & 182 & 14,3 \\
\hline Incapacidade Temporária & 164 & 136 & 179 & 73 & 121 & 76 & 47 & 796 & 62,6 \\
\hline Incapacidade Parcial & 6 & 7 & 4 & 6 & 22 & 2 & 13 & 60 & 4,7 \\
\hline Incapacidade Total Permanente & - & 2 & 1 & - & 2 & 4 & 3 & 12 & 0,9 \\
\hline $\begin{array}{l}\text { Óbito por Acidente de Trabalho } \\
\text { Grave }\end{array}$ & 15 & 12 & 8 & 29 & 25 & 15 & 51 & 155 & 12,2 \\
\hline Óbito por Outras Causas & - & - & 1 & 1 & - & - & - & 2 & 0,2 \\
\hline Outro & 1 & - & - & 1 & - & - & 2 & 4 & 0,3 \\
\hline Ignorado & 6 & 11 & 6 & 12 & - & 10 & 15 & 60 & 4,7 \\
\hline
\end{tabular}

Fonte: Elaboração própria.

Nota: Sinal convencional utilizado:

- Dado numérico igual a zero não resultante de arredondamento.

Conforme visualizado na Figura 1, o estado apresentou tendência geral decrescente significativa na ocorrência de casos de acidente de trabalho grave. Foram realizados modelos de regressão linear simples para a taxa de incidência de acidentes, conforme mostrado na Tabela 1 . Apesar do coeficiente de determinação $(\mathrm{R} 2=0,58)$ não ser elevado, o seu respectivo p-valor $(0,03)$ mostrou-se significativo. 
Figura 1 - Frequência e incidência de acidentes de trabalho graves ocorridos e registrados por ano Sergipe, Brasil - 2009-2015

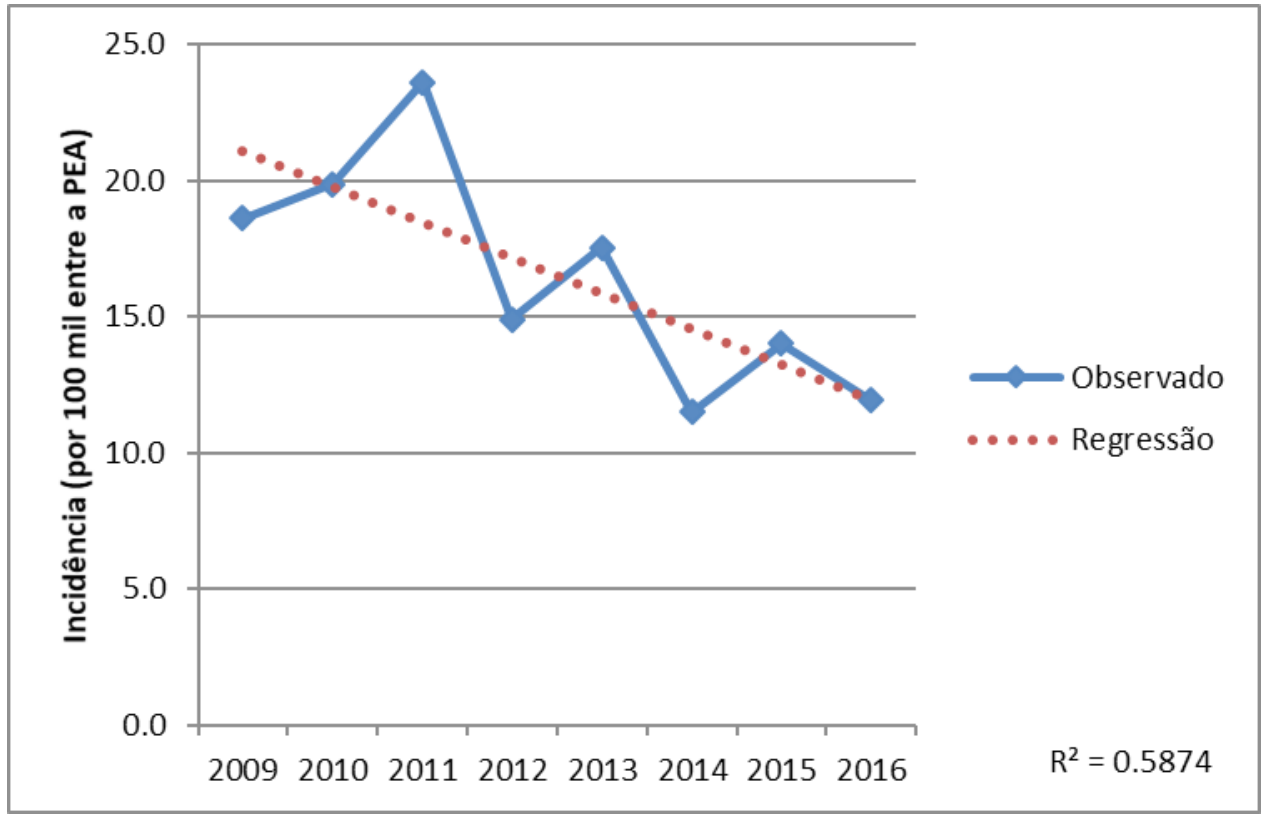

Fonte: Elaboração própria.

\section{Discussão}

Em Sergipe, no período compreendido entre 2009 e 2015 foi registrado um total de 1.271 acidentes de trabalho grave, o que proporciona uma média de 158,87 acidentes graves ao ano. Média relativamente alta, que reforça a necessidade de redução do número de acidentes, mediante estratégias específicas, como a Estratégia Nacional para Redução dos Acidentes do Trabalho 2015-2016, proposta pelo Ministério do Trabalho e Emprego ${ }^{(16)}$.

Analisando ano a ano, verificou-se a existência de uma considerada variabilidade na taxa de incidência desses acidentes (baseada na PEA), ao demonstrar crescente acometimentos em Sergipe nos anos de 2009 a 2011, sendo justamente o ano de 2011, o de maior taxa. Após esse crescente, são observadas flutuações, que são comuns na literatura sobre o tema. A maioria dos trabalhos também apresenta taxas variáveis, como em estudo realizado no Paraná ${ }^{(17)}$ e outro no Rio Grande do Norte ${ }^{(18)}$.

Além desses, na prática diária, seja no setor de saúde ou em outros setores, é possível visualizar a não uniformidade das taxas de acidentes, uma vez que essas são influenciadas por diversos fatores que diferem ao longo dos meses e dos anos.

Dos casos de acidente de trabalho grave analisados, a maioria foi do sexo masculino $(88,8 \%)$ e pertencente à faixa etária de adultos jovens entre 25 e 29 anos (17,1\%), o que também foi observado em vários outros estudos relacionados ao tema ${ }^{(19-21)}$. Esse fato é justificado pelo fato de os homens de menor faixa etária executarem tarefas de maior grau de risco para acidentes ${ }^{(22)}$.

Vale destacar que, apesar de este trabalho ter como critério de exclusão os acidentes ocorridos com menores de 16 anos, foi identificada a ocorrência de acidentes de trabalho grave em crianças e adolescentes - situação igualmente evidenciada em estudo do Rio Grande do Norte $^{(18)}$. Também cabe destacar que a taxa de acidentes na faixa de 25 a 29 anos foi pouco expressiva $(17,1 \%)$, em virtude de essa faixa etária ter sido agrupada em dez categorias, com predominância dos acidentes entre 16 e 39 anos, representando $68 \%$ da amostra, o que corrobora 
o predomínio dos acidentes entre os adultos em idade produtiva.

Este fato é bastante preocupante, já que boa parte dos acidentes leva à incapacidade temporária $(62,3 \%)$, de modo a afastar o trabalhador no início de sua carreira laboral, muitas vezes, gerando sequelas significativas que interferem diretamente no seu desempenho futuro ou mesmo levando à morte prematura.

A cor da pele/raça também foi analisada e demonstrou que os acidentes graves de trabalho, em Sergipe, aconteceram predominantemente na população parda (61,5\%), fato contrário ao achado em outro estudo ${ }^{(17)}$, em que a maior parte dos acidentados era de pele branca (70,3\%). Esta contradição pode ser justificada pela variação da distribuição dessa característica na população brasileira, tendo em vista que o destaque da população de acidentados brancos deu-se na Região Sul do país, local em que muitas comunidades são descendentes de povos europeus.

Com relação à escolaridade, percebeu-se que os acidentes ocorreram primordialmente entre os menos qualificados. A maior parte não chegou ao ensino médio, com destaque para a faixa de estudo da $5^{\underline{a}}$ a $8^{\underline{a}}$ série incompleta. Alguns estudos também destacaram a pouca escolaridade entre os acidentados ${ }^{(22)}$. Estudo desenvolvido no Paraná ${ }^{(17)}$ revelou que $21 \%$ dos acidentados concluíram o ensino médio completo, o que mostra grau de escolaridade dos paranaenses maior quando comparado às outras regiões do país.

Quanto às características do acidente, constatou-se que 59,8\% dos acidentes foram típicos e $35,8 \%$ de trajeto, corroborando resultados semelhantes encontrados em outro estudo ${ }^{(23)}$. Cabe destaque para o crescimento dos acidentes de trajeto que, embora em menor número, atinge porcentagens semelhantes ao de acidente típico. A crescente violência nas ruas e, particularmente, no trânsito justifica esse crescimento ${ }^{(24)}$.

A CAT auxilia no cálculo dos encargos trabalhistas gerados para o empregador, isto é, quando mais a instituição emite CAT, maior a sua carga de tributos, constituindo-se em um dos motivos para a sua não emissão ${ }^{(25)}$. A CAT é uma ferramenta de grande importância para a epidemiologia dos acidentes de trabalho e a sua emissão é obrigatória. Neste estudo, entretanto, ela somente foi emitida em 9,6\% dos casos, sendo ignorada na maior parte deles (36\%), não se identificando as possíveis causas que geraram esse fato.

Além disso, a CAT não registra os acidentes com os trabalhadores informais; dessa forma, é o SINAN um importante banco de informações epidemiológicas sobre os acidentes, já que engloba todos os trabalhadores: os formais e os informais. Embora os dados disponibilizados pela Previdência Social tenham como referência a situação do trabalhador brasileiro, sabe-se que esse registro muitas vezes não é feito.

O regime de tratamento adotado no atendimento dos acidentados foi principalmente $\mathrm{O}$ hospitalar $(57,9 \%)$, justificado por tratar-se de acidentes graves, que normalmente têm como direcionamento os hospitais grandes de média e alta complexidade.

A avaliação da tendência mostrou-se decrescente ao longo dos anos analisados e previu uma incidência de 11,9 para o ano de 2016. A tendência foi significativa para a ocorrência de casos de acidente de trabalho grave na série temporal analisada, com p-valor $(0,03)$. Resultados semelhantes foram vistos pelo Ministério do Trabalho e Emprego ${ }^{(14)}$, em estudo de tendência realizado entre os anos de 2003 e 2013, em que, apesar de ainda se apresentarem como números expressivos, há uma diminuição do quantitativo de acidentes.

\section{Conclusão}

Os resultados deste estudo revelam uma tendência geral decrescente dos acidentes de trabalho graves no estado de Sergipe durante o período estudado. Embora esse decréscimo tenha demonstrado significância, ainda é elevado o número de acidentes graves de trabalho no estado com o seguinte perfil: trabalhadores do sexo masculino, em idade produtiva, pardos e de baixa escolaridade que sofreram, principalmente, acidentes típicos, que necessitaram de 
regime hospitalar e resultaram, em sua maioria, em incapacidades temporárias.

O conhecimento da tendência é de grande importância para a formulação e estruturação de programas de prevenção e para a elaboração de estratégias mais específicas e eficientes. A temática do acidente de trabalho demanda a atenção de profissionais de diversas áreas, de núcleos distintos, em especial, os da medicina e os da enfermagem do trabalho, que possam tratar dos riscos e das possibilidades de prevenção, por meio de uma abordagem interdisciplinar.

Mais estudos devem ser realizados na região, para identificar a ocorrência dos fatores intrinsicamente relacionados aos acidentes graves de trabalho, e fazer uma análise mais rigorosa da estrutura de funcionamento da rede de assistência à saúde. Para que haja redução significativa desses incidentes graves, é importante que mais estudos possam auxiliar com a identificação precoce e institua intervenções efetivas para diminuir essas taxas.

\section{Colaborações:}

1 - concepção, projeto, análise e interpretação dos dados: Ana Caroline Rodrigues Lima, Damião da Conceição Araújo e Julianne Souza Bezerra;

2 - redação do artigo e revisão crítica relevante do conteúdo intelectual: Jadiel Fellipe Santana Santos e Jéssica Oliveira da Cunha;

3 - aprovação final da versão a ser publicada: Ana Caroline Rodrigues Lima e Allan Dantas dos Santos.

\section{Referências}

1. Martins JC, Pires JM. O trabalho humano na América Latina: evolução histórica e condições atuais. Cad PROLAM/USP. 2015;14(27):11-25. DOI: 10.11606/issn.1676-6288.prolam.2015.110375

2. Miranda FMD'A, Cruz EDA, Félix JCV, Kalinke LP, Mantovani MF, Sarquis LMM. Profile of Brazilian workers victims of occupational accidents with biological fluids. Rev Bras Enferm. 2017 Oct;70(5):1061-8. DOI: 10.1590/ 0034-7167-2016-0482
3. Prates D, Silva AE. Interruptions of activities experienced by nursing professionals in an intensive care unit. Rev Latino-Am Enfermagem. 2016;24:e2802. DOI: 10.1590/1518-8345.0997.2802

4. Batista AG, Santana VS, Ferrite S. The recording of fatal work-related injuries in information systems in Brazil. Ciênc Saúde Coletiva. 2019 Mar;24(3):693704. DOI: 10.1590/1413-81232018243.35132016

5. Santos SVMS, Macedo FRM, Silva LA, Resck ZMR, Nogueira DA, Terra FS. Work accidents and selfesteem of nursing professional in hospital settings. Rev Latino-Am Enfermagem. 2017;25:e2872. DOI: $10.1590 / 1518-8345.1632 .2872$

6. Mejia CR, Cárdenas MM, Gomero-Cuadra R. Notificación de accidentes y enfermedades laborales al Ministerio de Trabajo. Perú 2010-2014. Rev perú med exp salud publica [Internet]. 2015 [cited 2019 Oct 16];32(3):526-31. Available from: http://www.scielo.org.pe/scielo.php?script=sci_ arttext\&pid=S1726-46342015000300018

7. Instituto Nacional do Seguro Social. Manual de Acidente de Trabalho [Internet]. Brasília; 2016. [cited 2019 Oct 16]. Available from: http://file.abiplast.org.br/download/2016/ manualdeacidentedetrabalhoinss2016.pdf

8. Ribak S, Oliveira EJN, Rosolino GP, Orru PN, Tietzmann A. Epidemiology of traumatic injuries of the upper limbs in a university hospital. Acta ortop bras. 2018;26(6):370-3. DOI: 10.1590/1413-785220182606180607

9. Batista AG, Santana VS, Ferrite S. Registro de dados sobre acidentes de trabalho fatais em sistemas de informação no Brasil. Ciênc Saúde Coletiva. 2019 Mar;24(3):693-704. DOI: 10.1590/1413-81232018243.35132016

10. Takala J, Hämäläinen P, Saarela KL, Yun LY, Manickam K, Jin TW, et al. Global estimates of the burden of injury and illness at work in 2012 . J Occup Environ Hyg. 2014;11(5):326-37. DOI: $10.1080 / 15459624.2013 .863131$

11. Malta, DC, Stopa SR, Silva MMA, Szwarcwald CL, Franco MS, Santos FV, et al. Acidentes de trabalho autorreferidos pela população adulta brasileira, segundo dados da Pesquisa Nacional de Saúde, 2013. Ciênc Saúde Coletiva. 2017;22(1):169-78. DOI: 10.1590/1413-81232017221.17862015

12. Brasil. Ministério do Trabalho e Emprego. Estratégia Nacional para Redução dos Acidentes do Trabalho 2015-2016. Brasília; 2015. 
13. Organização das Nações Unidas. OIT: Mais de 313 milhões de trabalhadores sofrem acidentes de trabalho todos os anos [Internet]. Brasília; 2015 [cited 2019 Oct 19]. Available from: https:// nacoesunidas.org/oit-mais-de-313-milhoes-detrabalhadores-sofrem-acidentes-de-trabalhotodos-os-anos/

14. Instituto Brasileiro de Geografia e Estatística. Cidades@ Sergipe [Internet]. Rio de Janeiro; 2016 [cited 2019 Oct 16]. Available from: http://cidades. ibge.gov.br/xtras/uf. php?coduf $=28$

15. Instituto Brasileiro de Geografia e Estatística. Estatística dos indicadores de Trabalho e Rendimento [Internet]. Rio de Janeiro; 2016 [cited 20 Oct 2019]. Available from: http:// www.ibge.gov.br/home/estatistica/indicadores/ trabalhoerendimento/pme/pmemet2.shtm

16. Drumond EF, Silva JM. Avaliação de estratégia para identificação e mensuração dos acidentes de trabalho fatais. Ciênc Saúde Coletiva. 2013;18(5):1361-5.

17. Scussiato LA, Sarquis LMM, Kirchhof ALC, Kalinke LP. Perfil epidemiológico dos acidentes de trabalho graves no estado do Paraná, Brasil, 2007 a 2010. Epidemiol Serv Saúde. 2013;22(4):621-30.

18. Cavalcante CAA, Medeiros SM, Mata MS, Cavalcante EFO, Cavalcante ES, Oliveira LV. Acidentes de trabalho grave no Rio Grande do Norte: estudo transversal. Online braz j nurs. 2015;14(4):543-55. DOI: https://doi.org/10.17665/1676-4285.20155221

19. Viegas Louise Raissa Teixeira, Almeida Milena Maria Cordeiro de. Perfil epidemiológico dos casos de LER/DORT entre trabalhadores da indústria no Brasil no período de 2007 a 2013. Rev bras saúde ocup. 2016;41:e22. DOI: http://dx.doi. org/10.1590/2317-6369000130615
20. International Labour Organization. Seoul declaration on safety and health at work. The Safety and Health Summit [Internet]. Seoul: ILO; 2008 [cited 2019 Oct 6]. Available from: http://www. ilo.org/wcmsp5/groups/public/@dgreports/@ dcomm/documents/statement/wcms_095910.pdf

21. Bortoleto MSS, Nunes EFPA, Haddad MCL, Reis GAX. Acidentes de trabalho em um pronto atendimento do Sistema Único de Saúde. Rev Espaço para Saúde. 2011;13(1):91-7. DOI: http:// dx.doi.org/10.22421/1517-7130.2011v13n1p91

22. Campos AG, Gurgel AM. Acidentes de trabalho graves e atividades produtivas nas regiões administrativas de saúde em Pernambuco: uma análise a partir da identificação de aglomerados produtivos locais. Rev bras saúde ocup [online]. 2016;41:e15. DOI: 10.1590/2317-6369000115215

23. Miranda FMD, Scussiato LA, Kirchhof ALC, Cruz EDA, Sarquis LMM. Caracterização das vítimas e dos acidentes de trabalho fatais. Rev Gaúcha Enferm. 2012;33(2):45-51.

24. Cordeiro R, Luz VG, Hennington ÉA, Martins ACA, Tófoli LF. Urban violence is the biggest cause of fatal work-related accidents in Brazil. Rev Saúde Pública. 2017 Dec;51:123. DOI: 10.11606/ s1518-8787.2017051000296

25. Batista AG, Santana VS, Ferrite S. Registro de dados sobre acidentes de trabalho fatais em sistemas de informação no Brasil. Ciênc Saúde Coletiva. 2019;24(3):693-704. DOI: 10.1590/1413-81232018243.35132016

Recebido: 17 de outubro de 2019

Aprovado: 24 de outubro de 2019

Publicado: 5 de fevereiro de 2020

A Revista Baiana de Enfermagem utiliza a Licença Creative Commons - Atribuição-NãoComercial 4.0 Internacional. https://creativecommons.org/licenses/by-nc/4.0/

Este artigo é de acesso aberto distribuído sob os termos da Licença Creative Commons (CC BY-NC). Esta licença permite que outros remixem, adaptem e criem a partir do seu trabalho para fins não comerciais. Embora os novos trabalhos tenham de lhe atribuir o devido crédito e não possam ser usados para fins comerciais, os usuários não têm de licenciar esses trabalhos derivados sob os mesmos termos. 